\title{
ANALYTIC SOLUTIONS OF NONLINEAR NEUTRAL AND ADVANCED DIFFERENTIAL EQUATIONS
}

\author{
JOSEPH WIENER \\ DEPARTMENT OF MATHEMATICS \\ PAN AMERICAN UNIVERSITY \\ EDINBURG, TEXAS 78539 \\ LOKENATH DEBNATH \\ DEPARTMENT OF MATHEMATICS \\ UNIVERSITY OF CENTRAL FLORIDA \\ ORLANDO, FLORIDA 32816 \\ and \\ S.M. SHAH \\ DEPARTMENT OF MATHEMATICS \\ UNIVERSITY OF KENTUCKY \\ LEXINGTON, KENTUCKY 40506 \\ (Received May 15, 1985)
}

ABSTRACT. A study is made of local existence and uniqueness theorems for analytic solutions of nonlinear differential equations of neutral and advanced types. These results are of special interest for advanced equations whose solutions, in general, lose their margin of smoothness. Furthermore, existence of entire solutions is established for linear advanced differential systems with polynomial coefficients.

KEY WORDS AND PHRASES. Existence and uniqueness of holomorphic solutions, Nonlinear neutral and advanced differential equations.

1980 AMS SUBJECT CLASSIFICATION CODES. 34Al0, $34 \mathrm{~A} 34$.

1. INTRODUCTION.

The well-known Izumi theorem [1] states that if in the equation

$$
w^{(n)}(z)+a_{1}(z) w^{(n-1)}\left(\lambda_{1}(z)\right)+\ldots+a_{n}(z) w\left(\lambda_{n}(z)\right)=b(z) \quad(1.1)
$$

$a_{i}(z), b(z), \lambda(z)$ are regular in the disk $|z| \leq 1$ and $\lambda_{i}(0)=0,\left|\lambda_{1}(z)\right|<1$, for $|z| \leq 1$, there exists a unique solution with the given $w^{(1)}(0)$ regular in the closed disk $|z| \leq 1$. In a recent paper, Cooke and Wiener [2] have generalized this result for linear neutral equations with infinitely many arguments. Pelyukh [3] has studied some nonlinear neutral equations of the Izumi type.

This paper is concerned with the study of local existence and uniqueness theorems for analytic solutions of nonlinear neutral and advanced differential equations. A theorem on entire solutions of linear advanced differential equations is also provided. 
2. HOLOMORPHIC SOLUTIONS OF NONLINEAR NEUTRAL EQUATIONS.

We consider the equation

$$
w^{\prime}(z)=f\left(z, w(z), w(\lambda(z)), w^{\prime}(\lambda(z))\right) \text {. }
$$

If $\lambda(z)$ has a fixed point $z_{0}$, then an initial value problem for (2.1) can be posed at $z_{0}$ in the same manner as for ordinary differential equations. We may always assume $z_{0}=0$, that is, $\lambda(0)=0$ and prescribe for $(2.1)$ an initial value $w(0)=w_{0}$. Putting $z=0$ in (2.1) gives the equation

$$
w^{\prime}(0)=f\left(0, w_{0}, w_{0}, w^{\prime}(0)\right)
$$

for the unknown value $w^{\prime}(0)$.

THEOREM 1. As suume for (2.1) the following hypotheses:

(i) Equation $(2.1)$ has a solution $w^{\prime}(0)=w_{0}^{\prime}$.

(ii) The function $f\left(z, w, w_{1}, w_{2}\right)$ is holomorphic in the region $\mathrm{R}:|\mathrm{z}| \leq \mathrm{r}_{0}, \quad\left|\mathrm{w}-\mathrm{w}_{0}\right| \leq \mathrm{M}_{0},\left|\mathrm{w}_{1}-\mathrm{w}_{0}\right| \leq \mathrm{M}_{0},\left|\mathrm{w}_{2}-\mathrm{w}_{0}^{\prime}\right| \leq \mathrm{M}_{1}$, where $\mathrm{M}_{1} \geq \frac{\mathrm{M}_{0}}{\mathrm{r}_{0}}+\left|\mathrm{w}_{0}^{\prime}\right|$, and satisfies a Lipschitz condition

$$
\begin{aligned}
& \left|f\left(z, w, w_{1}, w_{2}\right)-f\left(z, y, y_{1}, y_{2}\right)\right| \\
& \leq L_{0}|w-y|+L_{1}\left|w_{1}-y_{1}\right|+L_{2}\left|w_{2}-y_{2}\right|,
\end{aligned}
$$

where $\mathrm{L}_{2}<1$.

(iii) The function $\lambda(z)$ is holomorphic in the disk $|z| \leq r_{0}$ and satisfies in it the inequality $|\lambda(z)| \leq|z|$.

Then in some disk $|z| \leq r$ there exists a unique holomorphic solution of equation (2.1) with the initial values $w_{0}$, $w_{0}^{\prime} \cdot$

PROOF. We replace $(2.1)$ by the integral equation

$$
w(z)=w_{0}+\int_{0}^{z} f\left(s, w(s), w(\lambda(s)), w^{\prime}(\lambda(s))\right) d s
$$

and introduce the operator

$$
\operatorname{Tg}(z)=w_{0}+\int_{0}^{z} f\left(s, g(s), g(\lambda(s)), g^{\prime}(\lambda(s))\right) d s
$$

on the space $G$ of all functions $g(z)$ holomorphic in the disk $|z| \leq r$ and satisfying the conditions.

$$
g(0)=w_{0}, g^{\prime}(0)=w_{0}^{\prime},\left|g(z)-w_{0}\right| \leq \frac{r_{0}}{r_{0}} .
$$

The value of $r$ is to be determined later. Clearly, the first restriction on $r$ is $r \leq r_{0}$. Since $g^{\prime}(z)-w_{0}^{\prime}$ is the derivative of $\left(g(z)-w_{0}\right)-w_{0}^{\prime} z$, we have

$$
\left|g^{\prime}(z)-w_{0}^{\prime}\right| \leq \frac{M_{0}}{r_{0}}+\left|w_{0}^{\prime}\right|
$$

in $|z| \leq r$. Taking in hypothesis (ii)

$$
M_{1} \geq \frac{M_{0}}{r_{0}}+\left|w_{0}^{\prime}\right|,
$$

we conclude that the function $f\left(z, g(z), g(\lambda(z)), g^{\prime}(\lambda(z))\right.$ ) is holomorphic (and 
bounded) in this disk. Let

$$
M(r)=\max \left|f\left(z, g(z), g(\lambda(z)), g^{\prime}(\lambda(z))\right)\right|, \quad|z| \leq r .
$$

Then from (2.3),

$$
\left|\mathrm{Tg}(\mathrm{z})-\mathrm{w}_{0}\right| \leq \mathrm{M}(\mathrm{r})|\mathrm{z}| \leq \mathrm{r} \mathrm{M}(\mathrm{r}) \text {. }
$$

We choose $r$ such that $r M(r) \leq r M_{0} / r_{0}$, that is

$$
M(r) \leq M_{0} / r_{0}
$$

which is always possible to do. Now, we evaluate

$$
\begin{aligned}
\left|\frac{d}{d z} \operatorname{Tg}(z)-w_{0}^{\prime}\right| & =\left|f\left(z, g(z), g(\lambda(z)), g^{\prime}(\lambda(z))\right)-w_{0}^{\prime}\right| \\
& \leq\left|f\left(z, g(z), g(\lambda(z)), g^{\prime}(\lambda(z))\right)\right|+\left|w_{0}^{\prime}\right| \\
& \leq M(r)+\left|w^{\prime}{ }_{0}\right| \leq \frac{M_{0}}{r_{0}}+\left|w^{\prime}{ }_{0}\right| \leq M_{1},|z| \leq r .
\end{aligned}
$$

In $R$ the function $f$ satisfies a Lipschitz condition

$$
\begin{aligned}
& \left|f\left(z, w, w_{1}, w_{2}\right)-f\left(z, y, y_{1}, y_{2}\right)\right| \\
& \leq L_{0}|w-y|+L_{1}\left|w_{1}-y_{1}\right|+L_{2}\left|w_{2}-y_{2}\right| \text { with } L_{2}<1 .
\end{aligned}
$$

We next introduce a metric in the space $G$ by the formula

$$
d\left(g_{1}, g_{2}\right)=\left(L_{0}+L_{1}\right) \max \left|g_{1}(z)-g_{2}(z)\right|+L_{2} \max \left|g_{2}^{\prime}(z)-g_{2}^{\prime}(z)\right|,|z| \leq r .
$$

Then, from $(2.3)$,

$$
\begin{aligned}
& \left|\operatorname{Tg}_{1}(z)-\operatorname{Tg}_{2}(z)\right| \leq L_{0} r \max \left|g_{1}(z)-g_{2}(z)\right| \\
& +L_{1} r \max \left|g_{1}(\lambda(z))-g_{2}(\lambda(z))\right|+L_{2} r \max \left|g_{1}^{\prime}(\lambda(z))-g_{2}^{\prime}(\lambda(z))\right| \\
& \leq\left(L_{0}+L_{1}\right) r \max \left|g_{1}(z)-g_{2}(z)\right|+L_{2} r \max \left|g_{1}^{\prime}(z)-g_{2}^{\prime}(z)\right|
\end{aligned}
$$

and

$$
\max \left|\mathrm{Tg}_{1}(z)-\mathrm{Tg}_{2}(z)\right| \leq \mathrm{rd}\left(\mathrm{g}_{1}, \mathrm{~g}_{2}\right)
$$

Furthermore,

$$
\begin{aligned}
\left|\frac{d}{d z} \operatorname{Tg}_{1}(z)-\frac{d}{d z} \operatorname{Tg}_{2}(z)\right| & =\mid f\left(z, g_{1}(z), g_{1}(\lambda(z)), g_{1}^{\prime}(\lambda(z))\right) \\
& -f\left(z, g_{2}(z), g_{2}(\lambda(z)), g_{2}^{\prime}(\lambda(z))\right) \mid \leq d\left(g_{1}, g_{2}\right)
\end{aligned}
$$

and

$$
\max \left|\frac{d}{d z} \operatorname{Tg}_{1}(z)-\frac{d}{d z} \operatorname{Tg}_{2}(z)\right| \leq d\left(g_{1}, g_{2}\right)
$$

Multiplying $(2.5)$ by $\left(\mathrm{L}_{0}+\mathrm{L}_{1}\right)$ and $(2.6)$ by $\mathrm{L}_{2}$ and adding yields

$$
\mathrm{d}\left(\mathrm{Tg}_{1}, \mathrm{Tg}_{2}\right) \leq\left(\mathrm{r}\left(\mathrm{L}_{0}+\mathrm{L}_{1}\right)+\mathrm{L}_{2}\right) \mathrm{d}\left(\mathrm{g}_{1}, \mathrm{~g}_{2}\right)
$$


Finally, the condition

$$
\mathbf{r}<\left(1-\mathrm{L}_{2}\right) /\left(\mathrm{L}_{0}+\mathrm{L}_{1}\right)
$$

shows that $T$ is a contraction of the space $G$ into itself. This proves the theorem. 3. HOLOMORPHIC SOLUTIONS OF NONLINEAR ADVANCED EQUATIONS.

The equation (see Shah and Wiener [4])

$$
w^{\prime}(z)=a_{0} w(\lambda z)+a_{1} z w^{\prime}(\lambda z)+a_{2} z^{2} w^{\prime \prime}(\lambda z)
$$

is of considerable interest. If the coefficients are real and $0<\lambda<1$, then for $z>0$ it is of advanced type. Furthermore, it appears that advanced equations, in general, lose their margin of smoothness, and the method of successive integration shows that after several steps to the right from the initial interval the solution may not even exist. Nonetheless, (3.1) admits analytic solutions. Namely, if $0<|\lambda|<1$, then the initial-value problem $w(0)=w_{0}$ for the complex differential equation (3.1) with complex constants $a_{i}$ and $\lambda$ has $a$ unique holomorphic solution, and it is an entire function of zero order. In fact, substituting the series

$$
w(z)=\sum_{n=0}^{\infty} w_{n} z^{n}
$$

in (3.1) yields

$$
\begin{aligned}
\sum_{n=0}^{\infty}(n+1) w_{n+1} z^{n}=\sum_{n=0}^{\infty} a_{0} \lambda^{n} w_{n} z^{n}+\sum_{n=0}^{\infty} & (n+1) a_{1} \lambda^{n} w_{n+1} \\
& +\sum_{n=0}^{\infty}(n+2)(n+1) a_{2} \lambda^{n} w_{n+2} z^{n+2}
\end{aligned}
$$

and

$$
(n+1) w_{n+1}=\left(a_{0} \lambda^{n}+n a_{1} \lambda^{n-1}+n(n-1) a_{2} \lambda^{n-2}\right) w_{n}, \quad n \geq 0 .
$$

From here, it follows that for large $n$,

$$
\left|w_{n+1} / w_{n}\right| \leq c q^{n}
$$

with some constant $c$ and $q<1$.

A nonlinear analogue of $(3.1)$ is the equation

$$
w^{\prime}(z)=f\left(z, w(z), w(\lambda(z)), z w^{\prime}(\lambda(z)), \ldots, z_{w}{ }^{(n)}(\lambda(z))\right),
$$

$$
w(0)=w_{0} \cdot
$$

THEOREM 2. As sume that the function $f\left(z, w, w_{\lambda}, w_{1}, w_{2}, \ldots, w_{n}\right)$ is holomorphic in

$$
R:|z| \leq r_{0},\left|w-w_{0}\right| \leq M_{0},\left|w_{\lambda}-w_{0}\right| \leq M_{0},\left|w_{i}\right| \leq M_{1},(i=1, \ldots, n) .
$$

and $\lambda(z)$ is holomorphic in the disk $|z| \leq r_{0}$ and satisfies in it the inequality $|\lambda(z)| \leq|z|$. 

(3.2).

Then in some disk $|z| \leq r$ there exists a unique holomorphic solution of problem PROOF. Replace (3.2) by the integral equation

$$
w(z)=w_{0}+\int_{0}^{z} f\left(s, w(s), w(\lambda(s)), s w^{\prime}(\lambda(s)), \ldots s w^{n} n(\lambda(s))\right) d s
$$

and introduce the operator

$$
\operatorname{Th}(s)=w_{0}+\int_{0}^{z} f(s, h(s)) d s
$$

where

$$
h(s)=\left(g(s), g(\lambda(s)), s^{\prime}(\lambda(s)), \ldots, s^{n} g^{(n)}(\lambda(s))\right),
$$

on the space $H$ of all functions $g(z)$ holomorphic in the disk $|z| \leq r$ and satisfying the conditions

$$
g(0)=w_{0},\left|g(z)-w_{0}\right| \leq m, m \leq M_{i} / 1 !,(i=0, \ldots, n) .
$$

The first restriction on $r$ is $r \leq r_{0}$. Since $g^{(i)}(z)$ is the derivative of order $i$ of the function $g(z)-w_{0}$, we have

$$
\left|z^{i}{ }^{(i)}(z)\right| \leq i ! m \leq M_{i}, \quad(i=1, \ldots, n)
$$

for $|z| \leq r$. Therefore, the function $f(z, h(z))$ is holomorphic in this disk. Let $M=\max \left|f\left(z, w, w_{\lambda}, w_{1}, \ldots, w_{n}\right)\right|$ in $R$. Then, from (3.3)

$$
\left|\operatorname{Th}(z)-w_{0}\right| \leq M r \text {, }
$$

and we choose $r$ such that $M r \leq M_{0}$, that is $r \leq M_{0} / M$. Furthermore,

$$
\left|\frac{d^{i}}{d z^{i}} T h(z)\right|=\left|\frac{d^{i-1}}{d z^{i-1}} f(z, h(z))\right| \leq \frac{(i-1) ! M}{r^{i-1}}, \quad i \geq 1
$$

and

$$
\left|z^{i} \frac{d^{i}}{d z^{i}} \operatorname{Th}(z)\right| \leq(i-1) ! M r
$$

The requirement $(i-1) ! M r \leq M_{i}$ gives $r \leq \frac{M_{i}}{(1-1) ! M}$ for $1 \leq 1 \leq n$. In $R$ the function $f$ satisfies a Lipschitz condition

$$
\begin{aligned}
& \left|f\left(z, w, w_{\lambda}, w_{1}, \ldots, w_{n}\right)-f\left(z, y, y_{\lambda}, y_{1}, \ldots, y_{n}\right)\right| \\
& \leq L_{0}|w-y|+L_{\lambda}\left|w_{\lambda}-y_{\lambda}\right|+\sum_{i=1}^{n} L_{i}\left|w_{i}-y_{i}\right| .
\end{aligned}
$$

We introduce a metric in the space $H$ by the formula

$$
\begin{aligned}
d\left(h_{1}, h_{2}\right)=\left(L_{0}+L_{\lambda}\right) \max & \left|g_{1}(z)-g_{2}(z)\right| \\
& +\sum_{i=1}^{n} L_{i} \max \left|z^{i}\left(g_{1}^{(i)}(z)-g_{2}^{(i)}(z)\right)\right|,|z| \leq r .
\end{aligned}
$$

Then, from (3.3) 


$$
\max \left|\operatorname{Th} h_{1}(z)-T h_{2}(z)\right| \leq r d\left(h_{1}, h_{2}\right) .
$$

Furthermore,

$$
\begin{aligned}
& \max \left|z^{i} \frac{d^{i}}{d z^{i}}\left(T h_{1}(z)-T h_{2}(z)\right)\right| \\
& =\max \left|z^{i} \frac{d^{i-1}}{d z^{i-1}}\left(f\left(z, h_{1}(z)\right)-f\left(z, h_{2}(z)\right)\right)\right| \\
& \leq r^{i} \frac{(i-1) !}{r^{i-1}}|\max | f\left(z, h_{1}(z)\right)-f\left(z, h_{2}(z)\right) \mid \\
& \leq(i-1) ! r d\left(h_{1}, h_{2}\right),(i=1, \ldots, n) .
\end{aligned}
$$

Multiplying (3.4) by $\left(L_{0}+L_{\lambda}\right)$ and (3.5) by $L_{i}$ and adding all inequalities yields

$$
d\left(T h_{1}, T h_{2}\right) \leq\left[r\left(L_{0}+L_{\lambda}\right)+\sum_{i=1}^{n}(i-1) ! L_{i}\right] r d\left(h_{1}, h_{2}\right) .
$$

Finally, if

$$
\left(L_{0}+L_{\lambda}\right)+\sum_{i=1}^{n}(i-1) ! L_{i}<1,
$$

then $T$ is a contraction of the space $H$ into itself, which proves the existence and uniqueness for (3.2).

REMARKS. Theorem 2 holds true if on the right of (3.2) the terms $z^{j_{w}(j)}(\lambda(z))$ are changed to $z^{k_{w}(j)}(\lambda(z))$, with $k_{j}>j$.

4. ENTIRE SOLUTIONS OF LINEAR SYSTEMS.

We are concerned with the equation

$$
W^{\prime}(z)=\sum_{i=0}^{M} \sum_{j=0}^{N} P_{i j}(z) W^{(j)}\left(\lambda_{i j} z\right), \quad W(0)=W_{0}
$$

in which $P_{i j}(z)$ and $W(z)$ are $d x d$ matrices.

THEOREM 3. As sume that $\mathrm{P}_{i j}(z)$ are polynomials of degree not exceeding $p$ :

$$
P_{i 0}(z)=\sum_{k=0}^{p} P_{i 0 k} z^{k}, P_{i j}(z)=\sum_{k=j-1}^{p} P_{i j k} z^{k},
$$

$$
(\mathrm{j} \geq 1, \mathrm{p} \geq \mathrm{N}-1),
$$

the complex numbers $\lambda_{i j}$ satisfy $0<\left|\lambda_{i j}\right|<1$ and the matrices

$$
B_{n}=(n+1) E-\sum_{i=0}^{M} \sum_{j=1}^{N} \frac{(n+1) !}{(n-j+1) !} \lambda_{i j}^{n-j+1} P_{i j, j-1}
$$

are nonsingular for all $\mathrm{n} \geq 0$, where $\mathrm{E}$ is the identity matrix. Then (4.1) has a unique holomorphic solution 


$$
W(z)=\sum_{n=0}^{\infty} W_{n} z^{n}
$$

and it is an entire function of zero order.

PROOF. From $(4.3)$ and $(4.2)$ we obtain

$$
\begin{aligned}
& W^{(j)}(z)=\sum_{n=0}^{\infty} \frac{(n+j) !}{n !} w_{n+j} z^{n}, \\
& W^{(j)}\left(\lambda_{i j} z\right)=\sum_{n=0}^{\infty} \frac{(n+j) !}{n !} \lambda_{i j}^{n} w_{n+j} z^{n}, \\
& P_{i j}(z) W^{(j)}\left(\lambda_{i j} z\right)=\sum_{k=0}^{p} P_{i j k} z^{k} \sum_{m=0}^{\infty} \frac{(m+j) !}{m !} \lambda_{1 j}^{m} W_{m+j} z^{m} \\
& =\sum_{n=0}^{\infty} z^{n} \sum_{s=0}^{n} \frac{(n-s+j) !}{(n-s) !} \lambda_{i j}^{n-s} P_{i j s} W_{n-s+j} .
\end{aligned}
$$

Since (4.2) implies $P_{i j s}=0$, for $s \leq j-2$, the index $s$ in the last sum extends from $j-1$ to $n$. Hence, the substitution $k=s-j+1$ leads to the equation

$$
(n+1) W_{n+1}=\sum_{i=0}^{M} \sum_{j=0}^{N} \sum_{k=0}^{n-j+1} \frac{(n-k+1) !}{(n-k-j+1) !} \lambda_{i j}^{n-k-j+1} \quad P_{1 j, k+j-1} W_{n-k+1} .
$$

From here,

$$
W_{n+1}=B_{n}^{-1} \sum_{i=0}^{M} \sum_{j=0}^{N} \sum_{k=1}^{n-j+1} \frac{(n-k+1) !}{(n-k-j+1) !} \lambda_{i j}^{n-k-j+1} P_{i j, k+j-1} W_{n-k+1,}, \quad n \geq 0 .
$$

Let $\left\|w_{n}\right\|=c_{n}$, then

$$
c_{n+1} \leq\left\|B_{n}^{-1}\right\| \sum_{i=0}^{M} \sum_{j=0}^{N} \sum_{k=1}^{p-j+1} \frac{(n-k+1) !}{(n-k-j+1) !}\left|\lambda_{i j}\right|^{n-k-j+1}|| P_{1 j, k+j-1}|| c_{n-k+1} \text {, }
$$

since $P_{i j k}=0$ for $k>p$. Furthermore,

$$
\frac{(n-k+1) !}{(n-k-j+1) !} \leq n^{j}
$$

and for large values of $\mathrm{n}$ we have

$$
\frac{(n-k+1) !}{(n-k-j+1) !}\left|\lambda_{1 j}\right|^{n-k-j+1} \leq q^{n} \text {, }
$$

where $\left|\lambda_{i j}\right|<q<1$. Also,

$$
\| B_{n}^{-1}|| \leq \frac{1}{n+1}\left(E-\sum_{1=0}^{M} \sum_{j=1}^{N} \frac{n ! \lambda_{1 j}^{n-j+1}}{(n-j+1) !} P_{1 j, j-1}\right)^{-1} \text {, }
$$

and for large $n$,

$$
\| B_{n}^{-1}|| \leq \mu /(n+1),
$$

with some constant $\mu$.

Therefore,

$$
c_{n+1} \leq c q^{n} \sum_{k=1}^{p+1} C_{n-k+1}, \quad c=\text { const. }
$$


Denote

$$
M_{n}=\max c_{k}, \quad 0 \leq k \leq n,
$$

then

$$
C_{n+1} \leq C(p+1) q{ }^{n} M_{n}
$$

Since $C(p+1) q^{n} \leq 1$ for large $n$, it follows that $C_{n+1} \leq M_{n}$ and $M_{n+1}=M_{n}$. Hence, starting with some natural number $m$,

$$
M_{n}=M_{m}, \quad n \geq m .
$$

Successively applying this result to (4.4) yields

$$
\begin{aligned}
& C_{m+1} \leq c(p+1) q^{m_{m}}, \\
& C_{m+2} \leq C(p+1) q^{m+1} M_{m+1} \leq c^{2}(p+1)^{2} q^{m} q^{m+1} M_{m},
\end{aligned}
$$

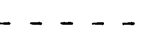

$$
\begin{aligned}
c_{m+n} & \leq c^{n}(p+1)^{n} q^{m} q^{m+1} q^{m+2} \cdots q^{m+n-1} M_{m} \\
& \leq c^{n}(p+1)^{n} q^{n(n-1) / 2} M_{m} \cdot
\end{aligned}
$$

This estimate for the coefficients $W_{n}$ concludes the proof. REMARKS. The strict inequalities $\left|\lambda_{1 j}\right|<1$ cannot be replaced by $\left|\lambda_{\mathbf{i j}}\right| \leq 1$

Indeed, the scalar equation

$$
w^{\prime}(z)=w(z)+\left(2 z-z^{2}\right) w^{\prime}(z), w(0)=w_{0}
$$

is of type (4.1), with $\lambda=1$. However, its solution

$$
w(z)=w_{0} e^{z /(1-z)}
$$

has a singularity at $z=1$.

\section{REFERENCES}

1. IZUMI, S., On the Theory of the Linear Functional Differential Equations, Tohoku Math. J. 30 (1929), 10-18.

2. COOKE, K. L. and WIENER, J., Distributional and Analytic Solutions of Functional Differential Equations, J. Math. Anal. App1. 98 (1984), 111-129.

3. PELYUKH, G. P., Existence and Uniqueness of $C^{r+1}$ - Solutions to Nonlinear Differential-Functional Equations of Neutral Type, Differential-Functional and Difference Equations, Kiev, 1981, 57-64 (In Russian).

4. SHAH, S. M. and WIENER, J., Existence Theorems for Analytic Solutions of Differential and Functional Differential Equations, Indian J. Pure and Appl. Math., to appear, (1986). 


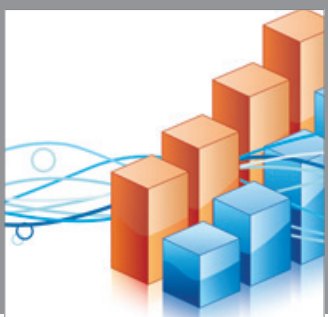

Advances in

Operations Research

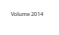

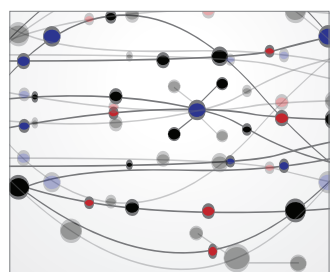

\section{The Scientific} World Journal
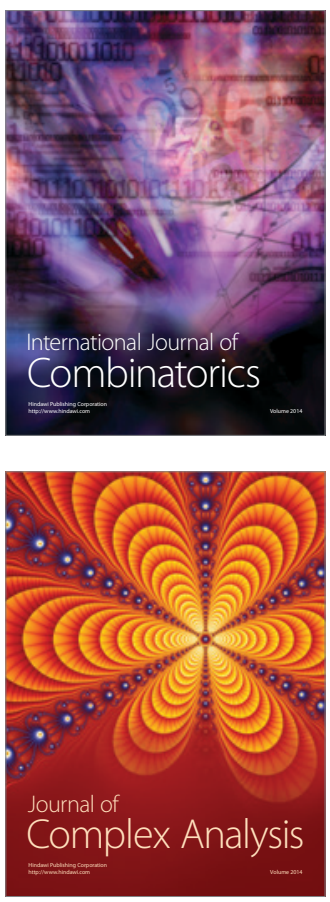

International Journal of

Mathematics and

Mathematical

Sciences
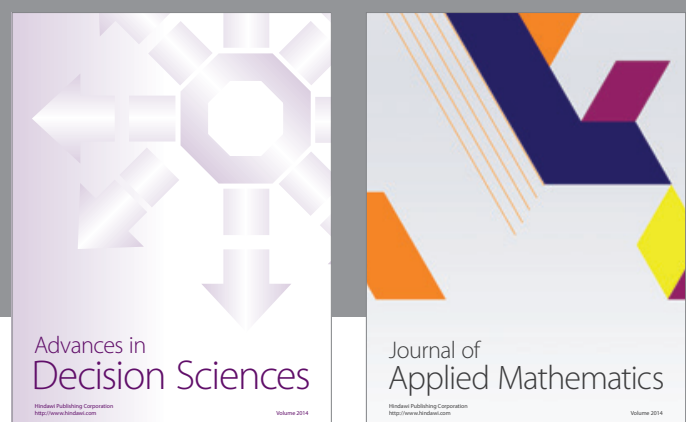

Journal of

Applied Mathematics
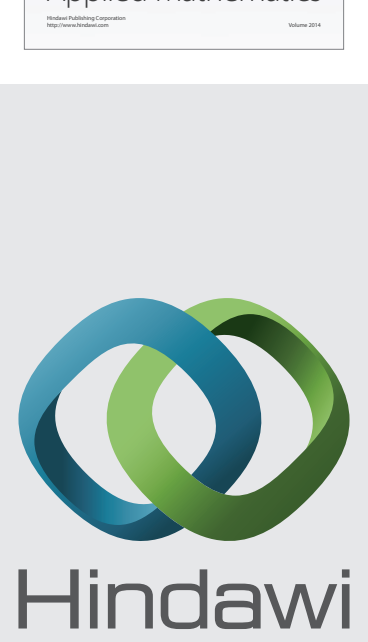

Submit your manuscripts at http://www.hindawi.com
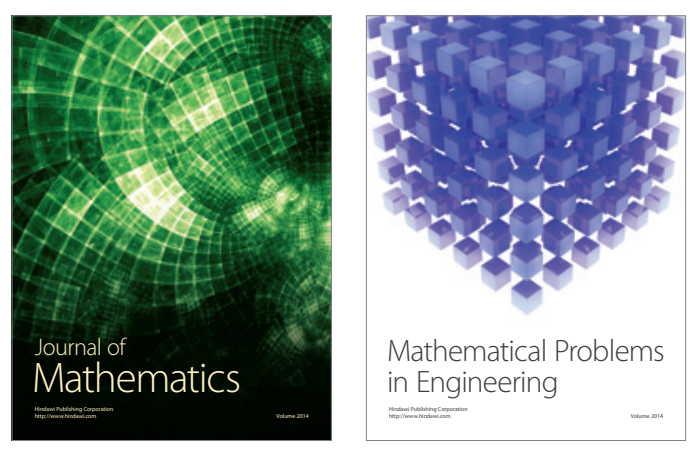

Mathematical Problems in Engineering
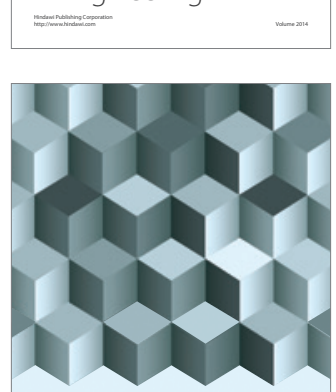

Journal of

Function Spaces
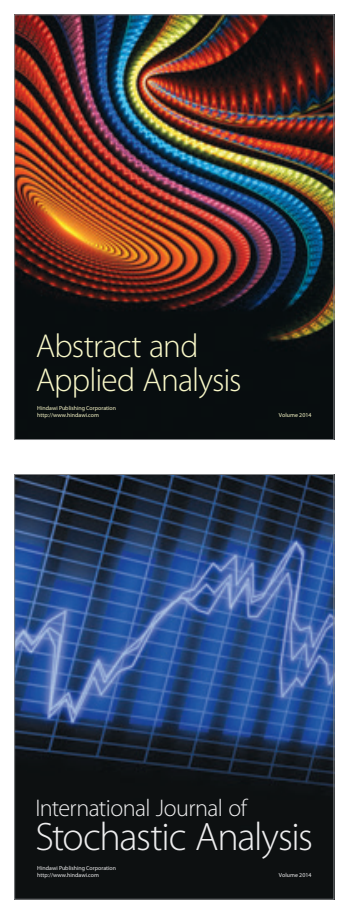

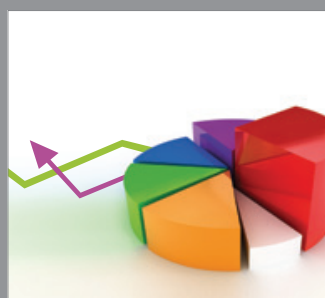

ournal of

Probability and Statistics

Promensencen
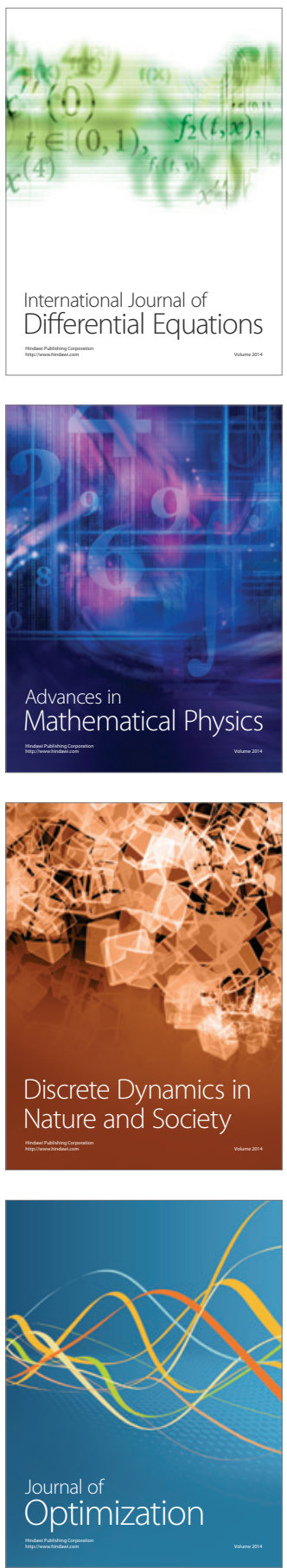